Fecha de recepción: octubre 2021

Fecha de aprobación: noviembre 2021

Fecha publicación: diciembre 2021

\section{La hibridación de la moda: la teoría del actor-red}

Ana María Ángeles Martínez Barreiro ${ }^{(1)}$

Resumen: En esta situación de pandemia global el sistema de la moda necesita más que nunca la constitución de un nuevo imaginario más sostenible y diferente. Donde la propuesta de Bruno Latour sobre la hibridez se enriquece con su teoría del actor red. El objetivo de este trabajo es desafiar el pensamiento convencional del estudio de la moda, a través de la teoría del actor red (ANT Actor-Network Theory) y la teoría de las prácticas sociales (TPS). Estos enfoques nos dan la oportunidad de ver las continuidades entre las prácticas de vestir y su amplio impacto ambiental y al mismo tiempo nos permite comenzar a pensar de forma más radical acerca de cómo nos conectamos con la naturaleza y la cultura, y de esta forma ensamblar las relaciones entre nuestra ropa y el medio ambiente.

Palabras clave: Moda - Naturaleza - Cultura - Sostenibilidad - Consumo - Actor-Red (ANT Actor-Network Theory) - Práctica Sociales (TPS) - Vestir - Medio Ambiente - Indumentaria.

[Resúmenes en inglés y portugués en las páginas 86-87]

(1) Ana Martínez Barreiro. Licenciada en Sociología, Universidade da Coruña. Doctora en Sociología, Universidade da Coruña. Directora del Departamento de Sociología y Ciencias de la Comunicación. Universidade da Coruña. Libros publicados: "La Moda en las sociedades Modernas". Publicaciones realizadas: "Crear Moda hacer Cultura: la cultura de la moda rápida" (2013), "La Cultura de usar y tirar: un problema de investigación" (2012), "Hacia un nuevo sistema de la moda: el modelo Zara" (2008). Co-directora del curso de Posgrado sobre la Moda: Diseño, Producción y Comunicación. Universidade da Coruña

\title{
Introducción
}

En esta situación de pandemia global el estudio de la moda necesita más que nunca la constitución de un nuevo imaginario más sostenible y diferente. Esta es una de las razones por las cuales este escrito va a caracterizar la naturaleza híbrida de la moda. Sin olvidar que indagar en la hibridación de moda significa pensar en una amplia gama de teorías y conceptos, que pueden ayudar a comprender la relevancia de la moda desde la teoría 
social y cultural, además de la cultura material de la ropa o la vestimenta y la propia sostenibilidad, (Bovone, 2015). De hecho, en los últimos años, las investigaciones en torno a la moda no han parado de crecer, hasta formar un campo de estudios de moda (Rocamora \& Smelik, 2016; Black et al., 2014, y Mora, et al., 2014a); y donde los estudios culturales han tenido un papel muy relevante en su desarrollo social, cultural, económico, al incorporar diversas disciplinas, como historia, sociología, antropología y los estudios de la mujer. Todos estos enfoques se encuentran en la unión de dos o más de las disciplinas mencionadas y han consiguiendo establecer una variedad de teorías y puntos de vistas diferentes desde la materialidad de la moda, la globalización, el consumo, la producción, los estudios postcoloniales, los estudios de género, los de comunicación y sostenibilidad. Si bien todos estos enfoques ofrecen muchas posibilidades de entender la moda, a través de su transversalidad. Sin embargo, no han calificado la moda como un híbrido entre la naturaleza y la cultura. A pesar de que nuestro mundo cotidiano siempre ha estado poblado de objetos híbridos. El primer autor que describe, la naturaleza híbrida de la moda es Barthes (1967), al distinguir entre lo material y lo ideal, en su obra el Sistema de la Moda, de matriz semiótica, y donde analiza la moda como un lenguaje.

El objetivo principal de este escrito es desafiar el pensamiento convencional de los estudios de la moda, a través de los estudios de ciencia y tecnología y su método asociado, la teoría del actor red (ANT Actor-Network Theory), esta última teoría ha conseguido plantear la investigación de la moda como un híbrido entre naturaleza y cultura. El concepto de hibridación ejemplifica aquellos procesos socio-culturales, ambientales y económicos en los que estructuras y prácticas que existen de forma separada, se combinan para generar nuevas estructuras, y objetos (Latour, 2007). Esta nueva perspectiva nos puede dar la oportunidad de ver las continuidades entre las prácticas de vestir y su amplio impacto ambiental y al mismo tiempo nos permite comenzar a pensar de forma más radical acerca de cómo nos conectamos con la naturaleza y la cultura, y de esta forma tratar de asignar y ensamblar plenamente las relaciones entre nuestra ropa y el medio ambiente. La explicación de por qué, la naturaleza ha llamado tan poco la atención en los estudios de la moda y que apareciera tardíamente dentro de las ciencias sociales tradicionales, está en la propia división sutilmente trazada entre el mundo natural y el mundo social (Entwistle, 2015). La estrategia de la modernidad, de purificar los fenómenos hasta ubicarlos o bien en la naturaleza o bien en la cultura, no ha permitido advertir el tránsito permanente entre ambos dominios, cuando en el mundo cotidiano se conjugan en universos indiferenciados (Latour, 2007). De hecho, donde los actuales costos ambientales del sistema de la moda ejemplifican muy bien esta división de la naturaleza y el mundo social, cuyo resultado ha sido insuficientemente analizado por los estudios de moda al centrar su interés en las desigualdades sociales, en los estudios culturales y las prácticas de consumo de moda (Entwistle, 2015).

En lo que respecta al concepto de hibridación hemos de insistir que la moda no sólo es cultura, sino también es industria, no sólo trata del consumo, sino también de la producción (Entwistle, 2000); además, de ser una de las industrias más globalizadas, pues un solo producto abarca decenas de grupos de interés $y$ varios continentes (Gardetti, 2018). Tras la postmodernidad los estudios de moda acuerdan estructurar el concepto de moda como sistema o fashion system y no como un mero discurso estético o de desigualdad social. 
La perspectiva de la moda como sistema ya ha sido abordada Entwistle (2000); y más recientemente por la socióloga Kawamura al definirla como un "Sistema de instituciones, organizaciones, grupos, productores, consumidores, eventos y prácticas, todos los cuales contribuyen a la creación de moda, que es diferente al concepto de ropa o vestimenta" (Kawamura, 2005, p. 45).

La moda no es sólo cambiante y efímera en su naturaleza, sino que es un sujeto híbrido, que requiere el estudio de la interconexión entre la producción y el consumo; y que cualquier análisis de la misma debe tener en cuenta varios agentes, instituciones y prácticas que formen intersecciones para producirlas (Entwistle, 2000, p. 101).

A partir de esta introducción, el artículo se organiza en tres partes. En la primera, abordamos el distanciamiento intelectual entre naturaleza y cultura. Pues, hoy en día estamos asistiendo a un proceso de discusión teórica que duda de la validez y eficacia de diversas dicotomías acuñadas en la modernidad, tales como mente/cuerpo, hombre/mujer, naturaleza/cultura. Donde la moda sólo se ha preocupado del yo-sociedad o de su estructura en lugar de la naturaleza-cultura. En la segunda, vamos a indagar en el concepto de la cultura del usar y tirar. Este concepto tiene mucho ver con el distanciamiento intelectual entre la naturaleza y el mundo social. En el tercer lugar, vamos a examinar la Teoría del Actor- Red (ANT) y la Teoría de las Prácticas Sociales (TPS); ambos enfoques nos ofrecen algunas vías para investigar la relación de la naturaleza y cultura y además nos pueden proporcionar nuevas formas de conectar a los diferentes actores y las prácticas de consumo en la actualidad. En cuanto a la metodología, este escrito se fundamenta en el desarrollo teórico y contextual de las cuestiones planteadas anteriormente. Sin olvidar que indagar en la hibridación de moda significa pensar en una amplia gama de teorías y conceptos, que pueden ayudar a comprender la relevancia de la moda desde la teoría social y cultural, además de la cultura material de la ropa o la vestimenta y la propia sostenibilidad (Bovone, 2015).

\section{Naturaleza y Cultura}

Actualmente, muchos de los problemas sociales y económicos que se detectan en la mayoría de las sociedades occidentales abarcan cuestiones relativas a la naturaleza y a la cultura. Una de las cuestiones que más ha llamado la atención a los científicos sociales en el ámbito de la moda son los problemas vinculados al medio ambiente y al coste de las prácticas de producción, además del consumo de los recursos naturales como el agua en la producción de algodón y el costo del transporte de la ropa en busca de la mano de obra más barata. Además, de todos los problemas del consumo excesivo de ropa y la cantidad de desperdicio que creamos, con sus constantes altos costos ambientales. El enfoque ecológico de la moda está personificado por varios académicos como Fletcher (2008); Gwilt \& Rissanen (2011); Salcedo (2014) y otros muchos que han abordado este tema desde diferentes perspectivas y en distintos ámbitos de interés. No obstante, en los años 60 del siglo pasado, el 
club de Roma elaboró un informe Los límites del crecimiento alertando que los índices de crecimiento industrial no son compatibles con la capacidad que tiene el planeta de soportar el crecimiento demográfico, la contaminación y el propio agotamiento de los recursos naturales (Meadows, et al; 1974). De ahí, la siguiente pregunta ¿En qué medida algunas de estas formas de entender la cuestión naturaleza-cultura han influido en las prácticas tan insostenibles del sistema de la moda? Bajo este enfoque trataremos de dar algunas respuestas a esta cuestión.

En primer lugar, el auge y prestigio alcanzado por las ciencias naturales en el siglo XIX impactó fuertemente en la configuración del saber. Esto trajo como consecuencia que el desarrollo de la producción científica del conocimiento estuviera distribuido en disciplinas diferentes. Esta estructuración del saber científico colocó a la sociología y a la antropología en un lugar de privilegio para el tratamiento de los temas que atañen a la cultura, pero al mismo tiempo reforzó los límites con los dominios de la naturaleza. La sociología y la antropología como disciplinas desde sus inicios se interesaron por los modos en que los distintos colectivos sociales interactúan con su entorno, pero dentro de los límites establecidos por la dicotomía naturaleza-cultura. Para Latour (2007), la modernidad, desde sus inicios, implicó prácticas de traducción y de purificación que llevaron a la comprensión de la realidad en forma dicotómica; entre otras cuestiones, indujo a separar a nivel filosófico y epistemológico a la naturaleza de la cultura.

En segundo lugar, la manera de comprender esta relación dicotómica entre naturaleza y el mundo social ha influido mucho en la construcción y perspectivas sobre la relación de las sociedades con la naturaleza. De hecho, bajo el desarrollo del capitalismo, la naturaleza es concebida como una materialidad útil para el progreso del hombre; una materialidad que es dominada y mejorada por obra de la ciencia, la técnica y las instituciones. Incluso, no sólo se trató a los recursos naturales como una forma de construcción del poder económico y geopolítico; sino como una forma de organizar, justificar y clasificar a las propias culturas. El etnocentrismo de la cultura europea frente a las extras europeas ha sido innegable. El etnocentrismo, al ordenar la realidad social de forma jerárquica; lo que hizo es establecer criterios de superioridad e inferioridad respecto a las culturas y la moda. Las categorías que se emplean en los diferentes estilos están en relación con las formas de la moda europea y aún se asocian con el colonialismo de los grandes imperios e incluso se habla con la intención de esquematizar, moda oriental, étnica, exótica, es decir, otra con respecto a la occidental (Frisa, 2020).

En tercer lugar, la misma desconexión entre la naturaleza y cultura ha puesto evidencia el deterioro del medio ambiente y la explotación de las condiciones de vida de los trabajadores en los países en desarrollo; florecido una abundante literatura que insiste en denunciar estas prácticas de explotación laboral como por ejemplo la publicación No Logo de Klein (2000); Siegle (2011) y Cline (2014) que investigaron y documentaron las prácticas de trabajo esclavo detrás de varias marcas de la moda. El derrumbe del edificio Rana Plaza es uno de los accidentes más mortales en la historia de la industria mundial de la confección, pero lamentablemente sólo uno de una serie de accidentes de fábrica en Bangladesh, Pakistán y otros países productores de prendas de vestir (Lohmeyer \& Schüßler, 2017). No obstante, los primeros debates se remontan a mediados de la década de 1990, cuando el tema de la explotación infantil -por parte de algunos proveedores de la marca Niké- llamó la aten- 
ción de la sociedad occidental (Mora et al., 2014b). A partir de ese momento, distintas organizaciones no gubernamentales, como Greenpeace y Clean Clothes Compaign instaron a las marcas de moda a avanzar hacia modelos y prácticas comerciales más sostenibles. En el año 2013, Greenpeace solicitó a las grandes marcas de la moda francesa e italiana que explicaran qué medidas estaban tomando para garantizar que sus productos no estuvieran implicados en modo alguno en la destrucción de los bosques ni el uso de sustancias químicas peligrosas y las sometió a un cuestionario de veinticinco preguntas en torno a tres temas ambientales: políticas para la compra de pieles, para el papel del packaging y para la producción textil. El proyecto se denomina The Fashion Duel y el resultado es una clasificación basada en la escala de un semáforo que asigna un nivel a cada marca y pone en evidencia las más positivas, las que aún pueden hacer más y las que no están comprometidas. En cuarto lugar, esta misma problemática ha puesto en evidencia la necesidad de potenciar los estudios académicos que impulsen la relación entre moda y medio ambiente. Recientemente el London Collage of Fashion fundó en 2008 el Centre For Sustainable, dirigido por Dilys Williams, para provocar, desafiar y poner en discusión el statu quo de la moda, al proyectar soluciones transformadoras en equilibrio entre la ecología, la sociedad y la cultura. Entre sus miembros podemos mencionar a estudiosos como Sandy Black y Kate Fletcher, activista en el campo de moda sostenible y titular del proyecto Local Wisdom. Sin embargo, para que se estableciera el tema de la sostenibilidad, en los estudios de moda tuvimos que esperar a que se publicaran algunos trabajos, como por ejemplo The Reader of Fashion escrita por Welters y Lillethun (2011); y The handbook of Fashion studies escrito por Black, et al; (2013). Esta misma problemática ha sido caracterizada por (Mora, et al; 2014b; Bovone,2015); y más recientemente por Martínez-Barreiro (2021).

\section{La cultura del usar y tirar}

Gran parte del debate en torno al medio ambiente y al desarrollo económico tienen mucho que ver con las pautas de consumo de la sociedad occidental. El interés que despierta la cultura del usar y tirar está estrechamente ligado a transformaciones sociales profundas, cambios en el modo de producción y en las formas de relación, A continuación, explicamos algunas de las razones de porque la cultura del usar y tirar ha contribuido al distanciamiento intelectual entre naturaleza y cultura.

En primer lugar, no podemos obviar las tendencias sobre las que se asientan las sociedades de consumo maduro: un sistema productivo con una capacidad de crear y fabricar de forma casi ilimitada y a un coste moderado, cuyo fin no es solamente el de satisfacer las demandas, sino producirlas para reproducirse. La propia utilización de afinadas técnicas de venta y promoción para crear un sentimiento de necesidad en el consumidor y conseguir persuadir hacia la compra. La eficacia de la moda y la publicidad a la hora de promover conductas de consumo derrochadoras y dotar a los bienes de un valor simbólico que generalmente se pierde con el tiempo y, en todo caso, mucho antes de que se pierda su utilidad funcional. Estos factores se han visto agravados por el auge del modelo de negocio de moda rápida (Martínez-Barreiro, 2012). 
En segundo lugar, a partir de esas transformaciones el individualismo se convierte en uno de los principales atributos de la sociedad postmoderna formada por individuos cuya capacidad de querer o desear ha sido separada o alienada de ellos mismo (Bauman, 2007). Desde el momento en que el individuo utiliza los objetos de consumo como forma de identificarse ante los demás, como forma de manifestar públicamente su estilo de vida, el sistema comienza a dar salida a todo lo que produce y por eso se generalizan tendencias como la de usar y tirar. Esta tendencia de usar y tirar se refleja en todos los ámbitos de la vida, convirtiéndose en un elemento característico de la propia cultura de la moda rápida. Articulando el concepto moda basura para designar unas prendas baratas, de dudosa calidad y que irremediablemente acabarán en los vertederos al poco tiempo de haber sido adquiridas (Siegle, 2011).

En tercer lugar, hasta hace poco la durabilidad era una característica primordial en los productos y objetos. Pero con el paso del tiempo, la durabilidad se ha pasado de moda porque la producción masiva de bienes de consumo exige una venta también masiva de esos productos. En este punto, es primordial el papel que juegan, por un lado, la moda, que se define principalmente por su condición efímera y pasajera y, por otro, la publicidad, cuya función informativa ha sido progresivamente sustituida por una función persuasiva con objetivos puramente comerciales. Todo ello induce a que los consumidores compran cada vez más, pero también que estén dispuestos a deshacerse de los bienes que compran en un corto período de tiempo. Asistimos a un proceso en el que se estimula el paso sistemático de la categoría de los bienes duraderos a la de los perecederos y consumibles (Martínez- Barreiro, 2012).

En cuarto lugar, el consumismo instantáneo y la eliminación de sus elementos, están en perfecta sintonía con el sistema de la moda rápida. La mayoría de los objetos pierden rápidamente su lustre y su atractivo, y lo más probable es que terminen en la basura incluso antes de haber producido alguna satisfacción. Es lo que se denomina la cultura de lo desechable (Bauman, 2007). Aquí radica una de las paradojas del consumismo, por una parte, es necesario reactivar las economías de las sociedades desarrolladas, pero a su vez al ser un sistema basado en el consumo desmesurado tampoco resulta aconsejable.

Otra de las paradojas está en el valor de cada uno de las prendas de moda ya no radica tanto en sus virtudes sino en sus limitaciones; esto es, en su obsolescencia pre-diseñada que permite la renovación y el rejuvenecimiento de las nuevas tendencias, tras la superación de la temporalidad, las empresas de moda rápida se han estructurado para producir y entregar durante todo el año, los síntomas de la moda rápida son: velocidad, exceso y desperdicio (Bauman, 2007, p. 120).

En quinto lugar, Li- Edelkoort (2015) una de las principales analistas de tendencias a nivel mundial, en su manifiesto el Anti-Fashion decreta que la cultura de moda está muerta. Su tesis observa que la moda se ha convertido en un fenómeno aislado, que se está posicionando peligrosamente al margen de la sociedad, con el riesgo de perder el contacto con lo que ocurre en el mundo. Pues, desde el momento que muchas prendas se ofrecen más baratas que un sándwich la sociedad siente que algo está mal. ¿Cómo puede costar un par de euros una camiseta que hay que sembrar, cultivar, cosechar, peinar, hilar, tejer, cortar y 
coser, terminar, imprimir, etiquetar, envasar y transportar? Esta es una de las razones por las cuales Edelkoort afirma que la moda ya no le interesa, pero si le interesan la ropa y los objetos en su materialidad, aspecto que tiene que ver con la necesidad, que otros muchos autores comparten, de recuperar una dimensión más concreta, tangible y menos inmaterial de la cultura y de sus formas donde todo está virtualmente desmaterializado. La cultura material es hoy objeto de una atención creciente por parte de los estudios culturales y, en particular, de la sociología. Pero ese no fue siempre el caso. Durante mucho tiempo la sociología, hablando de cultura, se refirió principalmente al mundo abstracto de las ideas e ideologías, a la herencia escrita de un pueblo, a las ciencias y las artes. Laura Bovone, (2015) y Shophie Woodward et al; (2014) ponen el énfasis en los procesos de materialización, ya sea enfocándose en cosas de moda o en los materiales mismos. Asimismo, Frisa en su libro Formas de moda afirma que, "la moda, más que nunca necesita, de sustancia" (2020, p. 9). La situación de pandemia global ocasionada por el coronavirus no solo define nuestro presente, sino que también desafía nuestro futuro de maneras inciertas, no solo con cuarentenas, aislamientos y distanciamiento social sino en las propias formas de encarar nuestra relación con la moda. El próximo apartado apunta en este sentido.

\section{La hibridez de la moda: la teoría del actor red}

La misma propuesta de hibridez de Latour se enriqueció con su teoría del Actor-red (ANT). Uno de los aspectos centrales de esta teoría es que plantea la investigación de la moda como un híbrido entre la naturaleza y la cultura (Entwistle, 2015; 2016). Lo cual nos permite ver las continuidades entre las prácticas de vestir y su amplio impacto ambiental y, al mismo tiempo, nos da la oportunidad de empezar a pensar de forma diferente sobre cómo conectamos la naturaleza y cultura. Y, al mismo tiempo, comenzar a ensamblar las relaciones sociales y culturales entre nuestra ropa y el medio ambiente (Entwistle, 2016). Para comprender el valor de este enfoque en los estudios de moda, hemos de tener en cuenta los siguientes principios, y la propuesta metodológica en la que se fundamenta la Teoría del Actor-Red.

En primer lugar, el enfoque de Latour (2007) ha conseguido desafiar algunas ideas preconcebidas sobre lo que es ser moderno, según él, la modernidad se contextualiza bajo la distinción artificial entre naturaleza y cultura y, a su vez, sostiene que el punto de vista de la cultura siempre se ha visto como superior y activa y por el contrario el de la naturaleza se la ve como inferior y pasiva. Y, el proponer es empezar a superar la creencia en la existencia de esferas delimitadas entre la naturaleza y la cultura pues no nos ha permitido advertir el tránsito permanente continuo entre ambos dominios, desde que habitamos un mundo donde naturaleza y cultura en nuestro cotidiano se conjugan en universos indiferenciados. En segundo lugar, Latour (2008) ha conseguido subrayar que los objetos que denominamos como naturales son en realidad productos histórico-sociales de nuestras formas particulares de ver el mundo, por lo tanto, son objetos o artefactos culturales. A partir de este supuesto, Latour (2007) considera que los objetos son simples híbridos de la relación entre la naturaleza-cultura. En esta misma línea, Latour \& Woolgar (1995) consideran que, de la 
misma forma, la ciencia moderna depende de algunos instrumentos que nos permiten ver cosas. Por ejemplo, los microscopios nos permiten ver las bacterias o células a simple vista; y esto ha llevado a la biología hacia las áreas de la cultura humana hasta llegar a examinar el genoma humano. Igualmente, Latour (2008) presupone que la naturaleza ya no puede ser vista como un área que esté ahí fuera o separada, y sea diferente de nosotros, sino que hemos de vernos de forma continua con la naturaleza, como una parte de ella, ya que nuestro parecer social basado en nuestra ropa y vestimenta es en realidad un híbrido de la relación entre la naturaleza y la cultura. El enfoque de Latour nos puede ayudar a abrir ese diálogo interno entre la ciencia y la cultura, entre la tecnología y la moda que algunos estudios han comenzado a investigar. En este sentido, la perspectiva del análisis del ciclo de vida (ACV) ha hecho algunos esfuerzos en el estudio del alargamiento de la vida de la ropa y los usos de la ropa (Fletcher \& Grose, 2012), además del estudio del impacto de los materiales y la propia huella ecológica que el sector de la moda produce (Choi, 2013). En cuarto lugar, siguiendo con la lógica de ver la moda como un sujeto híbrido entre la naturaleza y cultura; parece evidente que algo tan aparentemente cultural, como son las propias creaciones de moda, sea unas creaciones híbridas entre la naturaleza y la cultura. Las prendas son algo natural, puesto que están hechas por materiales naturales, como el algodón, la seda y el lino, y por otra parte, son montadas y ensambladas en nuestro mundo social y cultural de manera compleja, a través de las Cadenas Globales de producción textil (Gereffi y Korzeniewicz, 1994). De la misma forma, la teoría del sistema mundo (TSM) también nos puede servir como una herramienta muy útil para el análisis de la insostenibilidad de la moda rápida al permitir rastrear las conexiones entre las regiones periféricas donde se encuentra la mano de obra más barata y las zonas más desarrolladas (Wallerstein, 1974; Gereffi y Korzeniewicz, 1994). En este nuevo contexto ya no encajaría en absoluto hacer una clara distinción entre las dimensiones naturales y culturales de la moda, sino simplemente examinar el flujo continuo de materiales, objetos y actores que componen el sistema de la moda. Esto implicaría comenzar a pensar de forma más ecológica, es decir, vernos como parte de la ecología de la tierra y de ninguna manera separado o por encima de ella.

En quinto lugar, esta nueva forma de pensar depende de una comprensión diferente del significado de actor, ya que en esta teoría (ANT) los actores son tanto humanos como no humanos. En esta teoría un actor o actante es cualquier cosa con la capacidad de actuar de alguna manera; por ejemplo, el agua es un actor o actante, ya que es un componente activo de los materiales que componen de moda y, de hecho, casi todo lo demás también como el algodón, la seda, la lana, el lino, etc. La teoría del actor-red (ANT) es útil para ayudar a determinar cómo las categorías de personas y cosas que están en juego, interactúan en la moda. No obstante, por el principio de simetría (Callon, 1986), ninguno de los dos tendrá prioridad, ni será visto como una entidad separada o superior. Ya que la teoría del actor-red (ANT) rechaza la división ontológica de la materialidad y el significado, y la priorización del significado, en cambio, los materiales y las cosas se consideran una parte fundamental de los ensamblajes que llegan a constituir lo social (Latour, 2008). Por consiguiente, en este marco teórico, la moda se abordaría como un conjunto de elementos humanos y no humanos o actantes que componen el fenómeno de la moda en cuestión. Este enfoque ANT todavía no está muy bien desarrollado dentro de los estudios de moda, 
excepto en algunos estudios como el de Entwistle \& Slater (2014), que sí han tratado de investigar a las modelos dentro del sistema de la moda desde la perspectiva de la teoría de ANT.

En sexto lugar, la teoría del actor-red (ANT) también nos puede aportar una metodología para la localización de estas conexiones y nos puede enseñar a observar y a seguir a los actores, con el fin de ver dónde van y lo que hacen. Así pues, la teoría del actor-red no se trata de una teoría explicativa, sino descriptiva. Además, si utilizamos esta metodología sobre las prendas de moda, podríamos comenzar con los campos de algodón en la India, o las fábricas de producción en algún lugar de Asia, y seguir a los actores desde la producción de textiles a su ensamblado en prendas, su distribución a las tiendas y así llegar hasta los consumidores, e incluso ir mucho más allá del consumo, llegar hasta la eliminación de los residuos de ropa. Esto implica que, si llegamos a observar las prendas de ropa y las seguimos, podemos observar cómo los actores o actantes naturales entran en contacto con los agentes culturales, y se ensamblan en sistemas complejos o híbridos entre la naturaleza y la cultura. Por ejemplo, la producción de una prenda de vestir tiene mucho que ver con todos los procesos organizativos y productivo: los productos son seguidos a lo largo de su ciclo de vida mediante una trace mapping tool, implantada en el año 2008 por la empresa de los hermanos Rob y Martin Drake-Knight. Esta aplicación, que está disponible en el sitio web de la marca, permite identificar la posición geográfica exacta de los productos seleccionados, las procedencias y los lugares de extracción/creación para garantizar a los consumidores un acceso libre y transparente a las informaciones referentes a la cadena de distribución. Esta misma marca, Rapanui implantó, en colaboración con el Parlamento Europeo, un sistema de etiquetado ecológico para sintetizar la información relacionada con el packaging de las prendas y simplificar la compra consciente por parte de los consumidores. El proyecto es transformar el sistema de ecolabel en ley para toda la Unión Europea.

Por otra parte, la Teoría de las Prácticas Sociales (TPS) al hacer de la práctica su principal unidad de análisis, propone una aproximación totalmente distinta al desarrollar formas de explicación y de intervención en el área del comportamiento pro-ambiental, que van más allá de lo puramente motivacional o de los propios estilos de vidas (Bellotti y Mora; Klepp y Bjerk, 2014). Por ende, en el nuevo paradigma relacional de la moda debemos comenzar a prestar una cuidadosa atención a las prácticas de consumo sostenible y al propio diseño de la ropa. Algunos académicos ya han subrayado cómo el diseño se puede convertir en una herramienta muy útil para crear una vida más sostenible en un entorno social, cultural y físico, como ocurrió en el caso del propio diseño nordic walking (Pantzar \& Shove, 2005). Otros autores, desde el consumo, han abordado cómo las prácticas de usar y tirar la ropa pueden influir en el medio ambiente (Klepp \& Bjerk, 2014). En conclusión, el valor de este enfoque (TPS) dentro de la moda está, en primer lugar, en que estos estudios se basan más en el material físico que en el simbólico (Shove \& Pantzar, 2010), lo cual nos permite pensar en la moda sostenible como una práctica material y no solo como un mero discurso estético o de desigualdad social. En segundo lugar, estos estudios se presentan como un enfoque alternativo, al proponer el estudio de la relación de la ropa con otra ropa dentro de un todo mayor el armario como una forma de resaltar la materialidad de la ropa (Klepp \& Bjerk, 2014). Y, en tercer lugar, dentro de este enfoque la moda no se puede 
entender como un fenómeno separado o aislado, sino que está incrustada en prácticas culturales más amplias, y donde la sostenibilidad ya no puede ser vista como un complemento o algo separado de ella, de la moda o ropa; sino más bien como algo que surge de las propias prácticas cotidianas vestimentarias (Woodward \& Fisher, 2014).

\section{Conclusiones}

En primer lugar, este artículo no sugiere que debamos adoptar un enfoque exclusivamente (ANT) de la moda, sino que podemos aprender a pensar sobre diferentes facetas de la moda a través de sus múltiples elementos materiales y humanos y sus relaciones. Pensar en la moda como un híbrido entre naturaleza y cultura parece ser una perspectiva muy beneficiosa en el nuevo paradigma relacional de la moda.

En segundo lugar, hablar de moda es poner en evidencia la interrelación necesaria entre naturaleza y cultura. Y al mismo tiempo, descubrir una de las tantas combinaciones que permean nuestra sociedad, donde la pretensión de clasificar, categorizar y establecer límites precisos se vuelve cada vez más impracticable. En este nuevo contexto ya no encajaría en absoluto hacer una clara distinción entre las dimensiones naturales y culturales de la moda, sino simplemente examinar el flujo continuo de materiales, objetos y actores que componen el sistema de la moda.

En tercer lugar, la Teoría del actor red (ANT) ha conseguido plantear a la moda como un híbrido entre la naturaleza y la cultura. El entender la moda como un sujeto híbrido entre la naturaleza-cultura nos facilita ver las continuidades entre las prácticas de vestir y su amplio impacto ambiental y, al mismo tiempo, nos permite comenzar a pensar de una forma más radical acerca de cómo nos conectamos con la naturaleza y la cultura y, de esta forma, comenzar a ensamblar plenamente las relaciones entre nuestra ropa y el medio ambiente circundante.

En cuarto lugar, desde la teoría de las prácticas (TPS), la moda no se puede entender como un fenómeno separado o aislado, sino que está incrustada en prácticas culturales más amplias, y donde la sostenibilidad ya no puede ser vista como un complemento o algo separado de la moda o de la ropa, sino más bien como algo que surge de las propias prácticas cotidianas vestimentarias.

\section{Lista de Referencias Bibliográficas}

Bellotti, E. \& Mora, E. (2014). Networks of practices in critical consumption, Journal of Consumer Culture, 16 (3), 718-760. Barthes, R. (1967). Sistema de la moda. Barcelona: Gustavo Gili.

Black, S. (2008). Eco-Chic: The Fashion Paradox. Londres: Black Dog Publishing.

Black, S; Entwistle, E; Rocamora, A; De la haya, A; Root; R; Thomas, H. (2014). The Handbook of Fashion Studies. Londres, Reino Unido: Bloomsbury Publishing Plc. 
Bauman, (2007). Vida de consumo. Madrid: Fondo de cultura Económica.

Bovone, L. (2015). Cultura materiale e nuovi valori: il caso della moda etica. Sociología della comunicación, 50, 100-113.

Callon, M. (1986). Some elements of a sociology of translation: Domestication of the scallops and the fishermen of St Brieuc Bay. En: Law, J (ed.) Power, Action, and Belief: A New Sociology of Knowledge?. Londres: Routledge.

Choi, T. M. (2013). Local sourcing and fashion quick response system: The impacts of carbon footprint tax. Transportation Research Part E: Logistics and Transportation Review, (55), 43-54.

Cline, E. (2014). La moda desechable. El escandaloso costo de la moda rápida. Barcelona: Paidós.

Entwistle, J. (2000). The Fashioned Body. Cambridge: Polity Press.

Entwistle, J. (2015). Sustainability and fashion. En Fletcher, K. y Tham, M (eds.). Routledge Handbook of Sustainability and Fashion. Nueva York: Bloomsbury

Entwistle, J. (2016). Bruno Latour: Actor-Network- Theory and Fashion. En Rocamora, A; Smelik, A (eds). Thinking Through Fashion. Londres: I. B. Tauris Co.Ltd.

Entwistle, J. \& Slater, D. (2014). Reassembling the Cultural: fashion models, brands and the meaning of 'culture' after ANT. Journal of Cultural Economy, 7 (2), 161-177.

Fletcher, K (2008). Sustainable Fashion and Textiles. Design Journey. Earthscan.

Fletcher, K (2010). Slow fashion: an invitation for systems change. Fashion Practice: The Journal of Design, Creative Process and the Fashion, 2(2), 259-266.

Fletcher, K \& Grose, L (2012). Fashion \& Sustainability: Design for Change. Laurence King Publishing.

Frisa, María Luisa, (2020). Las formas de moda, cultura, industria, mercado. Buenos Aires: Ampersand.

Gardetti, M. (2018). Textiles y moda. ¿Qué es la sostenibilidad? Madrid: Lid.

Gereffi, G. \& Korzeniewicz, M. (1994). Commodity Chains and Global Capitalism. Westport: Greenwood Press.

Kawamura, Y. (2005). Fashion-ology: An Introduction to Fashion Studies. Oxford: Berg.

Klepp, I. G. \& Bjerck, M. (2014). A methodological approach to the materiality of clothing: Wardrobe studies. International Journal of Social Research Methodology, 17(4), 373- 386.

Latour, B. (2007). Nunca fuimos modernos, Ensayo de antropología simétrica. Buenos Aires, Argentina: Siglo XXI.

Latour, B. (2008). Reensamblar lo social. Una teoría a la introducción del actor red. Buenos Aires, Argentina: Manantial.

Latour, B. \& Woolgar, S. (1995). La vida en el laboratorio. La construcción de los hechos científicos. Madrid: Alianza Universidad.

Li- Edelkoort (2015). Anti-Fashion a manifesto for the next decade, in trendunion.

Lohmeyer, Nor \& Schüßler, Elke (2017). Rana Plaza as a threat to the fast fashion model? An analysis of institutional responses to the disaster in Germany. En: Becker-Leifhold, Carolina; Heuer, Mark; (eds.) Eco Friendly and Fair: Fast Fashion and Consumer Behavior. Sheffield: Greenleaf Publishing.

Martinez-Barreiro, A. (2012). La cultura del usar y tirar. ¿Un problema de investigación? Revista de Investigaciones Políticas y Sociales, (RIPS). 11 (4) 149-170. 
Martinez-Barreiro, A (2021). La sostenibilidad en los estudios de moda. Athenea Digital 21(1).

Meadows, Donella; Dennis. L. Meadows, D; Randers, J; Behrens.W; otros (1974). Los límites del crecimiento: informe del club de Roma sobre el predicament de la humanidad, México. Fondo de Cultura Económica

Mora. E; Rocamora, A; Volante, P. (2014 a). The internationalization of fashion studies: Rethinking the peer-reviewing process International Journal of Fashion Studies, 1 (1), 3-17.

Mora, E; Rocamora A; Volonté, P. (2014 b).On the issue of sustainability in fashion studies, International Journal of fashion Studies, 11 (2), 139-147.

Pantzar, M.\& Shove, E. (2005) Understanding innovation in practice. A discussion of the production and reproduction of Nordic Walking, Technology Analysis and Strategic Management, 22 (4), 447-462.

Rocamora, A \& Smelik, A. (eds) (2016). Thinking Through Fashion. Londres: I. B. Taurís. Salcedo, E. (2014). Moda ética para un futuro sostenible. Madrid: Gustavo Gili.

Shove, E. \& Pantzar, M. (2010). Consumers, producers and practices: understanding the invention and reinvention of Nordic Walking. Journal of Consumer Culture, 5 (1), 43-64.

Shove, E; Pantzar M; Watson, M. (2012). The Dynamics of Social Practice: Everyday Life and How it changes. Londres: Sage.

Siegle, L. (2011). To Die for: Is Fashion Wearing Out the World? Londres: Fourth Estate.

Skjerven, A. \& Reitan, J. (2017). Design for a Sustainable Culture: Perspectives, Practices and Education. Londres: Routledge.

Slater, K. (2003). Environmental Impact of Textiles: Production. Processes and Protection. Cambridge: Woodhead Publishing Limited/The Textile Institute.

Warde, A. (2005). Consumption and the theory of practice. Journal of Consumer Culture 5(2): 131-154.

Wallerstein, I. (1974). The modern world system. Nueva York: Academic Press, Berkeley: University of California Press.

Welters, L \& Lillethun, A. (2008- 2011). The Fashion Reader. 2nd edn. Oxford: Berg.

Woodward, S \& Fisher, T (2014). Introduction: Fashioning through materials: Material culture, materiality and processes of materialization. Critical Studies in Fashion \& Beauty, 5(1), 3-23.

\begin{abstract}
In this situation of a global pandemic, the fashion system needs more than ever the constitution of a new, more sustainable and different imaginary. Where Bruno Latour's proposal on hybridity is enriched with his theory of the actor network. The objective of this work is to challenge the conventional thinking of the study of fashion, through the actor network theory (ANT) and the theory of social practices (TPS). This approach gives us the opportunity to see the continuities between dress practices and their broad environmental impact and at the same time allows us to begin to think more radically about how we connect with nature and culture, and in this way assemble the relationships between our clothing and the environment.
\end{abstract}


Keywords: Fashion - Nature - Culture - Sustainability - Consumption - Actor-Network (ANT) - Social Practice (TPS).

Resumo: Nesta situação de pandemia global, o sistema da moda necessita mais do que nunca da constituição de um imaginário novo, mais sustentável e diferente. Onde a proposta de Bruno Latour sobre o hibridismo é enriquecida com sua teoria da rede de atores. O objetivo deste trabalho é desafiar o pensamento convencional do estudo da moda, por meio da teoria da rede de atores (ANT Ator-Rede Teoria) e da teoria das práticas sociais (TPS). Essas abordagens nos dão a oportunidade de ver as continuidades entre as práticas de vestuário e seu impacto ambiental mais amplo e, ao mesmo tempo, nos permitem começar a pensar mais radicalmente sobre como nos conectamos com a natureza e a cultura e, dessa forma, montamos. As relações entre nossos roupas e meio ambiente.

Palavras chave: Moda - Natureza - Cultura - Sustentabilidade - Consumo - Ator-Rede (ANT) - Prática Social (TPS).

[Las traducciones de los abstracts fueron supervisadas por el autor de cada artículo] 